\title{
Combining Ludology and Narratology in an Open Authorable Framework for Educational Games for Children: the Scenario of Teaching Preschoolers with Autism Diagnosis
}

\author{
Nikolas Vidakis ${ }^{1}$, Eirini Christinaki ${ }^{1}$, Iosif Serafimidis ${ }^{1}$, and Georgios Triantafyllidis ${ }^{2}$ \\ ${ }^{1}$ Technological Educational Institute of Crete, \\ Dept. Informatics Engineering, Heraklion, Crete, Greece \\ nv@ie.teicrete.gr, echrist@ics.forth.gr, sjosef23@gmail.com \\ ${ }^{2}$ Aalborg University, Medialogy Section, AD:MT, \\ Copenhagen, A.C.Meyers Vænge 15, Denmark \\ gtecreate.aau.dk
}

\begin{abstract}
This paper presents the initial findings and the on-going work of IOLAOS $^{1}$ project, a general open authorable framework for educational games for children. This framework features an editor, where the game narrative can be created or edited, according to specific needs. A ludic approach is also used both for the interface as well as for the game design. More specifically, by employing physical and natural user interface (NUI), we aim to achieve ludic interfaces. Moreover, by designing the educational game with playful elements, we follow a ludic design. This framework is then applied for the scenario of teaching preschoolers with autism diagnosis. Children with autism have been reported to exhibit deficits in the recognition of affective expressions and the perception of emotions. With the appropriate intervention, elimination of those deficits can be achieved. Interventions are proposed to start as early as possible. Computer-based programs have been widely used with success to teach people with autism to recognize emotions. However, those computer interventions require considerable skills for interaction. Such abilities are beyond very young children with autism as most probably they don't have the skills to interact with computers. In this context, our approach with the suggested framework employs a ludic interface based on NUI, a ludic game design and takes account of the specific characteristics of preschoolers with autism diagnosis and their physical abilities for customizing accordingly the narrative of the game.
\end{abstract}

\section{Introduction}

Increasingly, experts, teachers, parents and students look to technology as a complimentary support for their educations. Educational gaming is a great platform that helps in motivating students to learn and is designed to teach students about a specific subject and/or skills. Prensky in [1] argues that children are naturally motivated to

\footnotetext{
${ }^{1}$ IOLAOS in ancient Greece was a divine hero famed for helping with some of Heracles's labors.
} 
play games. Educational games are interactions that teach students goals, rules, adaptation, problem solving, interaction, all represented as a narrative. Such games give them the fundamental needs of learning by providing enjoyment, passionate involvement, structure, motivation, ego gratification, adrenaline, creativity, interaction and emotion. "Play has a deep biological, evolutionarily, important function, which has to do specifically with learning" [1].

In general, computer games and other digital technologies such as mobile phones and the Internet seem to stimulate playful goals and to facilitate the construction of playful identities. This transformation advances the ludification of today's culture in the spirit of Johan Huizinga's homo ludens [2]. In this context, this ludification of today's culture can be also used in educational activities to strengthen the motivation and the engagement of the students.

Moreover, the narrative of an educational game plays an important role in its success. The story is the root of the whole gaming experience. Up to now, educational games are usually created with a closed architecture and a single narrative, resulting to fail in providing a more personalized or customized learning procedure.

In this paper, we introduce the IOLAOS project, which is an open authorable framework for educational games for children. IOLAOS aims to combine ludology and narratology improvements to provide efficient educational gaming for children.

Regarding the game narrative, IOLAOS suggests a fully authorable editor (implemented in Unity game engine [3]), with which, experts can create templates and teachers can shape and customize the template-based games according to specific needs for a more personalized education. It's important that such customizations can be performed easily and without the reliance on software developers. The editor is also open. This means that new templates can be added easily for creating new games serving new educational goals.

Regarding the ludic approach, IOLAOS features the use of natural user interface. A natural user interface (NUI) is a human-computer interface that allows humans to communicate with the computer using standard modes of human communication such as speech or gestures, and to manipulate virtual objects in a fashion similar to the way humans manipulate physical objects. During the last few years, technology has been improved rapidly and allowed the creation of efficient and low-cost applications featuring natural user interfaces.

One of the characteristics of a successful natural user interface is thus the reduction of cognitive load on people interacting with it. This is an important feature that makes NUI a suitable interface in developing successful learning applications for children. In our design, NUI (instead of a restricted human-computer interface) is used to enhance playfulness and thus establish a ludic interface. NUI features and focuses also on the kinesthetic factor (gestures, movements, etc), which is an important element in achieving this playfulness of a ludic interface. For example, it is much more "fun" in a game to drive a car with your hands naturally, compared to pressing some key-board keys. And this is even more important and critical when the target group is children.

Besides the NUI-based interface, ludic design for the game has been also employed in order to improve playfulness, make the educational games more attractive for the children and aim to improve the learning procedure. 
Briefly, IOLAOS project:

- Introduces an open authorable narrative editor for creating templates and customizing educational games, without the reliance on software developers.

- Employs a twofold ludic approach for both the interface (NUI) and the game design.

- Aims to a creation of more personalized educational games that support the educational activities better.

As a proof of concept for the IOLAOS project, a work scenario is presented in this paper, for creating an education game for teaching preschoolers with autism spectrum conditions (ASC) to improve their skills in recognizing facial expressions. Facial expressions give important clues about emotions and provide a key mechanism for understanding, identifying and conveying them. Children with ASC often fail to recognize the qualitative differences and associations between various expressions of emotions [4]. Due to limited social and emotional understanding they do not know how to adequately interact with other people; a problem which sometimes leads to inappropriate behaviors. Studies have reported that individuals with ASC experience difficulties in recognizing expressions while in youth and experience problems recognizing emotions as adults [5].

Treatment approaches aim to improve social interaction, conquest communication and control inappropriate behavior. Children with ASC are more likely to initiate positive interaction after treatment [6]. Education is also considered as a solution for the socio-emotional deficits and training is claimed to improve face processing abilities and strategies in autism [7]. A variety of educational interventions have been proposed for children with autism and many proponents have claimed developmental improvement and other benefits [8].

In this context, this paper also presents how IOLAOS platform can be used in order to create an educational game featuring playfulness both in playing (NUI) and in designing the game, along with a customized narrative of the game, which can be edited according to the needs. Our aim is twofold, (a) to teach facial emotion recognition to preschoolers with ASC, and (b) to enhance their social interaction.

The rest of the paper is organized as follows. In section 2, a brief presentation of similar existing work in creating educational games is presented. Section 3 focuses on the proposed open architecture of the IOLAOS project. To illustrate the concepts of the proposed architecture, Section 4 presents the scenario for teaching preschoolers with ASC about expression recognition and how is this possible by using the IOLAOS framework. Finally, Section 5 describes conclusions and discusses future work.

\section{Background}

Educational games for children have been widely used in supporting learning in-side and out of school and as a result a growing interest has appeared for the potential of digital games to deliver effective and engaging learning experiences [9]. There is a 
variety of computer games and software that intend to assist users to achieve various educational goals. Well-known educational software is the project Scratch from MIT Media Lab [10], a programming language for learning to code. With Scratch users can program their own interactive stories, games and animations by putting together images, music and sounds with programming command blocks. Monterrat et al [11] in their study claimed that game moding as an educational activity could be interesting not only to learn programming but for any kind of learning. Their pedagogical tool allows people without game design skills to modify and share digital games. It allows a learner to become a teacher by designing an educational game that others can use to learn. Their main idea is that if learning a game helps students to acquire knowledge, then being able to change the game can provide students with the ability to deeply learn the content.

Narrative architecture and ludic design are two major approaches in contemporary video game theory. They both play important roles in teaching and learning as parts of educational gaming. Lester et al. [12] described the design issues and the empirical findings about motivation in narrative-centered learning environments. They found a strong connection between narrative and educational games and they claimed that narrative-centered learning environment is a promising approach for fostering positive learning gains, as well for promoting student motivation. On the other hand, PadillaZea et al. [13] included digital storytelling in an educational video game and introduced narrative elements to foster the students' motivation in learning processes by integrating specific educational models and ludic aspects. They claimed that ludic tasks in educational games are important elements to maintain students' interest, motivation and immersion.

During the last decade, researchers have begun to explore the use of computer technologies dedicated to ASC as intervention tools for improving and eliminating different deficits. In a recent review, Wainer and Ingersoll [14] examined innovation computer programs as educational interventions for people with ASC. They focused on studies describing programs to teach language, emotions or social skills. Their analysis showed that those tools are promising strategies for delivering direct intervention to individuals with ASC. Bernardini et al. [15] proposed a serious game for children with ASC to practice social communication skills; they used an intelligent virtual character that acts both as a peer and as a tutor on a number of different learning activities. These activities can be selected manually by a human operator (practitioner, parent or other carer) through a graphical interface. Their experimental results showed encouraging tendencies by relating the effectiveness of the children's interaction with the virtual character acting as a social partner to them. Porayska-Pomsta et al. [16] suggest an intelligent and authorable environment to assist children with ASC in gaining social interaction skills. Their tool contains an intelligent agent and a play environment that allows teachers and parents to become co-creators and tailor the game according to the needs of the individual children in their care. Although the design and creation of personalized games is crucial for children with ASC, as reported by the authors, limitations in the agent's intelligence (agent inability to deal with inappropriate or unexpected behavior from the user) contradicts the structured, stable and predictable learning environment that is also crucial. 
Ludology and narratology can also be considered as two important elements when creating educational games for children with ASC. Game narrative can provide context that assists children to apply the skills learned within the game. Ludology in both the interface and the game design also can engage children with autism in playful interactions and strengthen their motivation. Foster et al. [17] have suggested embedding interactive narrative in multimodal learning environments for social skill improvement of children with ASC. Castelhano et al. [18] studied therapeutic activities for children with developmental disabilities with the use of multisensory stimulation environments and documented its perception concerning ludic content, play and the computer-mediated ludic activity. The main theme that emerged from their study regarding playfulness was that the computer-mediated ludic experience is perceived as useful for intervention.

In general, educational computer games for children that combine ludology and narratology can provide an effective and engaging learning experience. Hence, developing learning environments that are both story-telling and play-based by combining narrative and ludicity may empower children to achieve great impact, improve deficits and gain new skills.

\section{The IOLAOS Platform}

The design of IOLAOS exhibits several novel characteristics, which differentiate an IOLAOS-based game from other forms of educational computer games and platforms. First of all, IOLAOS is not only concerned with educational computer games, but instead, it seeks to provide a guided learning environment for both educators and children, that is story-telling and play-based by combining narrative and ludic for harnessing knowledge. Consequently, its primary focus is to enable educators and children with the use of ludology and naratology to perform learning tasks and provide an effective and engaging learning experience. To achieve this, IOLAOS builds on a range of technologies, including semantic web, game engines and advanced human-computer interaction. Secondly, IOLAOS adopts a knowledge-based, reuseoriented and natural user interaction model to attain high quality during the performance of learning tasks.

\subsection{The Architecture}

The system architecture (figure1) consists of four distinct components that collaborate together to: (a) codify all different elements of educational theories and learning styles available and to create templates which are then offered to game developers, (b) compile games through a three step process, namely template customization, game creation and utilization definition, (c) manage learning session and play room attributes and (d) administer all necessary elements, users and their roles, game engine parameters etc. Peripheral to the system architecture are knowledge derived from educational theories, learning styles and classroom practices. The components of our architecture are the "Template Codifier", the "Game Compiler", the "Play Room" and the "System Administration". 


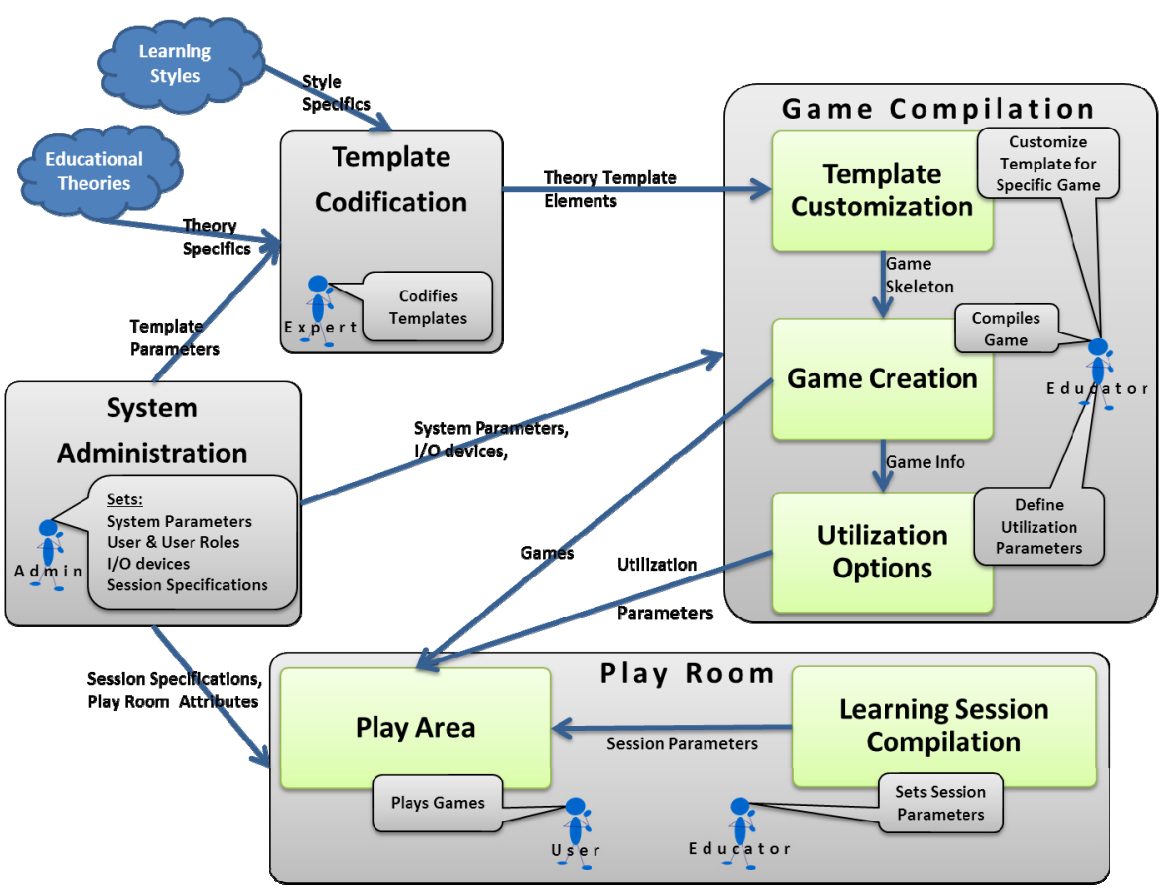

Fig. 1. System architecture

The "System Administration" component (see Figures $1 \& 2$ ) of the system is responsible for managing system attributes, template parameters, game elements, artifacts and behaviors, session attributes, input / output modalities, and user accounts and roles.

The "Template Codifier" component (see Figures $1 \& 3$ ) of the system is accountable for systemize / codify the various elements of the educational theories and learning styles. This is achieved by imprinting the theory's elements using a tabbed stepwise process by the expert. Apart from the first steps, that imprint basic information about the theories, the process has no strict order of step execution. The template codification process that has been developed in IOLAS in different tabs (see Figure 3) gives the user the capability to define the theory elements in an organized and clear manner. The educational theories and learning styles imprinting is performed by the role "Expert". The different groups of data that have been developed in IOLAOS for imprinting the theory's elements are: Template Basic Info, Style Basic Info, Target Group, Scenery Basics, Play Environment, Audio / Motion, Rewarding, Feedback, Evaluation (see Figure 3). 

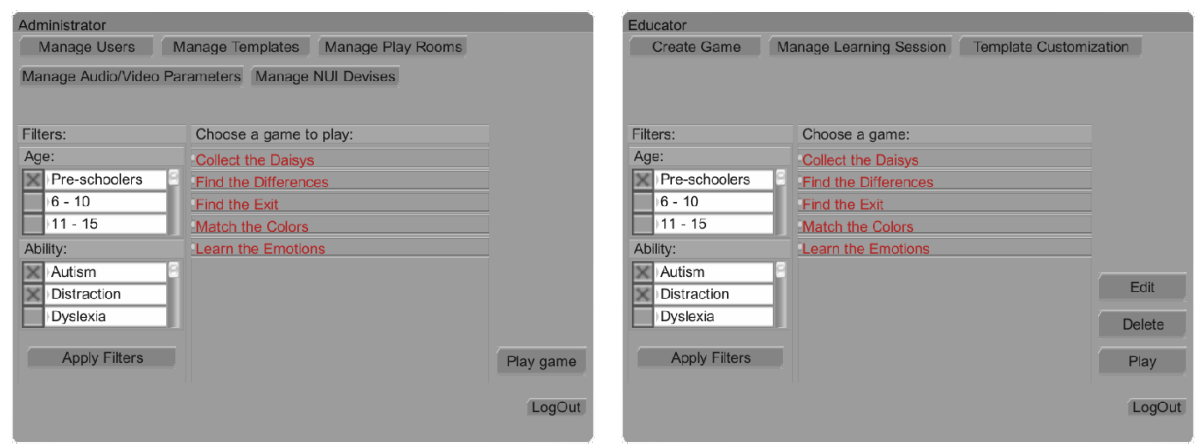

Fig. 2. IOLAOS administrator and educator main screens

In more detail, the "Template Basic Info" records general data such as: Title, Description, Theory base upon, Template Author, Creation Date etc. The "Style Basic Info" similarly records data concerning the learning style such as: Title, Description, Theory base upon, Template Author, Creation Date etc. The "Target Group" records data concerning player details, Abilities, and thematic areas such as: Age Group, School Grades, Thematic / Subject Area, Special Abilities etc. The "Scenery Basics" deals with data concerning the story telling that is involved in the game. Such data includes: Number of Scenes, Color Information, Texture, Narrative Criteria etc. The "Play_Environment" documents data with reference to the type of game (i.e. single player, small group, etc.), the environment played (i.e. supervised or not supervised) and the peripherals used (i.e. classic I/O devises, NUI devices, etc). The "Audio/Motion" records data concerning the use of sound and image input/output modalities such as Audio (yes, no, scalable), Motion (yes, no, number and frequency of moving artifacts) etc. The "Rewarding" deals with data concerning the rewarding of the player such as type of rewarding (i.e. textual, sound, movie, puzzle, etc.). The "Feedback" records all necessary information about feedback before, during and after the game flow (i.e. text, sound, movie, score, etc.). Finally the "Evaluation" deals with data concerning the evaluation of the player (i.e. evaluate per level or per game, or per game section etc.) as well as the evaluation type.

The "Game Compiler" component (see Figure 1) of the system consists of the "Template Customization" the "Game Creation" and the "Utilization Management". It is responsible for providing the "Educator" with the necessary tools to set up a ludic educational game. In other words, it gives the "Educator" the possibility to (a) customize the generic template set up by the "Expert" at the "Template Codification" component in such a way that suits the specific game requirements (see Figure 3) needed according to target user group abilities and educational goals to be achieved, (b) create a ludic game with the use of the tools provided by IOLAOS platform (c) to define game utilization parameters such as: Free Use, Registered User Only, etc. 


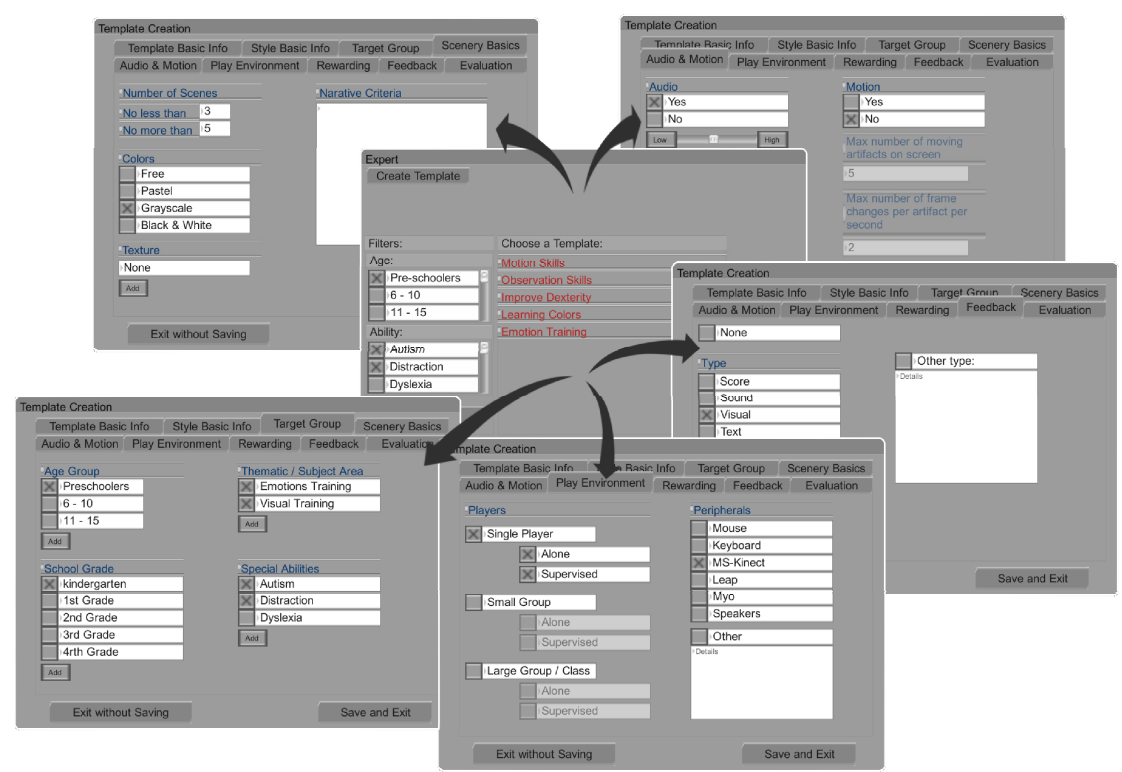

Fig. 3. The IOLAOS template codification and customization

Figures 3 exhibit selected elements of the individualized template for our representative scenario. In more detail we show that our game used a generic template for children with autism diagnosis and individualized it to fit the specified group abilities and educational goals, namely emotions recognition, set by the "Educator". The outcome is a Ludic Educational Game for preschoolers with special abilities and specific educational goals and is presented in detail in the next section.

The "Play Room" component (see Figure 1) of the system is responsible for setting up the appropriate space for playing games and consists of "Learning Session Compilation" and "Play Area".

The "Learning Session Compilation" gives "Educator" the ability to fully manage learning sessions according to individual, group or class requirements every time she/he needs to run an educational game. In specific "Educator" can determine: Players and/or Group, Marking / Evaluation Specifics / Procedure, Session Statistics, and Session parameters. She/he can also save incomplete learning sessions in order to be completed in the future.

The "Play Area" deals with game runtime specifics such as save, load, single player or multi player parameters etc.

The proposed architecture has been designed in order to support a game platform that fulfils the requirements of customized narratives, ludic interfaces and ludic game designing. The narrative is created by the expert and edited by the teacher according to learning needs and goals by using the template codification and template customization modules of the suggested architecture. The ludology is supported in two ways, first by creating and customizing ludic-based designed games through the template 
codification and game compilation components, and also by employing natural user interfaces to the playing process that enhance the playfulness of the educational game.

\section{Representative Scenario}

To illustrate some of the concepts described so far and to provide insight into the features of IOLAOS platform, we will briefly describe a representative scenario emphasizing on ludic, narrative and authorable game creation for educating children. Our reference scenario is summarized in Exhibit 1. For more details see [19].

Exhibit 1: Game begins with an instruction page where the child is informed what is going to happen, what he/she has to do and how he/she can do it. A two-hand gesture which is performed by moving both hands above the head is required to start the game. In the first level, children should learn labeling emotions by correlating emotion terms with images. The stimuli are presented on each trial with different pair of photos and the goal is to choose the correct image among the two. Selecting the left image (the orientation of the image is decided by looking toward the screen) requires a one-hand gesture which is performed by moving the left hand above the head. Image's color changes into light red for incorrect answer. Selecting the right image (the orientation again of the image is decided by looking toward the screen) requires a one-hand gesture which is performed by moving the right hand above the head. Image's color changes into light green for the correct answer. Moving to the next play area requires a two-hand gesture which is performed by moving both hands above the head. In the second level they should learn to recognize emotions from their description and their association with facial features. In the third level they should learn to identify the causes of various feelings in different situations, obtained through the use of social stories. At the end of the game, there is a congratulation message.

According to our reference scenario the "Educator" creates the game by performing the following steps in IOLAOS platform: (a) Select appropriate template, (b) Customize template according to scenario requirements, (c) Generate game framework upon which, the "Educator", will construct/fabricate the game, by defining artifacts and behaviors. The outcome of the above process is an educational game for children with autism diagnosis for emotion recognition.

In more detail, the "Educator" selects "Create Game" and at the "Select appropriate template" step he/she selects the appropriate template provided by IOLAOS, in our case the "Emotion Training" template. At step 2 ("Customize template according to scenario requirements") the "Educator" applies the scenario requirements which in our case are: 1) number of game levels are limited to 3 excluding welcome screen and final screen, 2) feedback is passed to player through light coloring of his choices (light red for wrong answer, light green for correct answer) during game execution and as concluding feedback at the end with the form of a congratulation message, and 3) Game navigation is performed via hand gestures with the use of MS-Kinect NUI device (raise left hand, right hand or both hands). At step 3 "Generate Game Framework" the platform allows the "Educator" to construct the game (see Figure 4) by using the artifacts and behaviors provided by IOLAOS according to his desires and the boundaries set up at step 2 . 


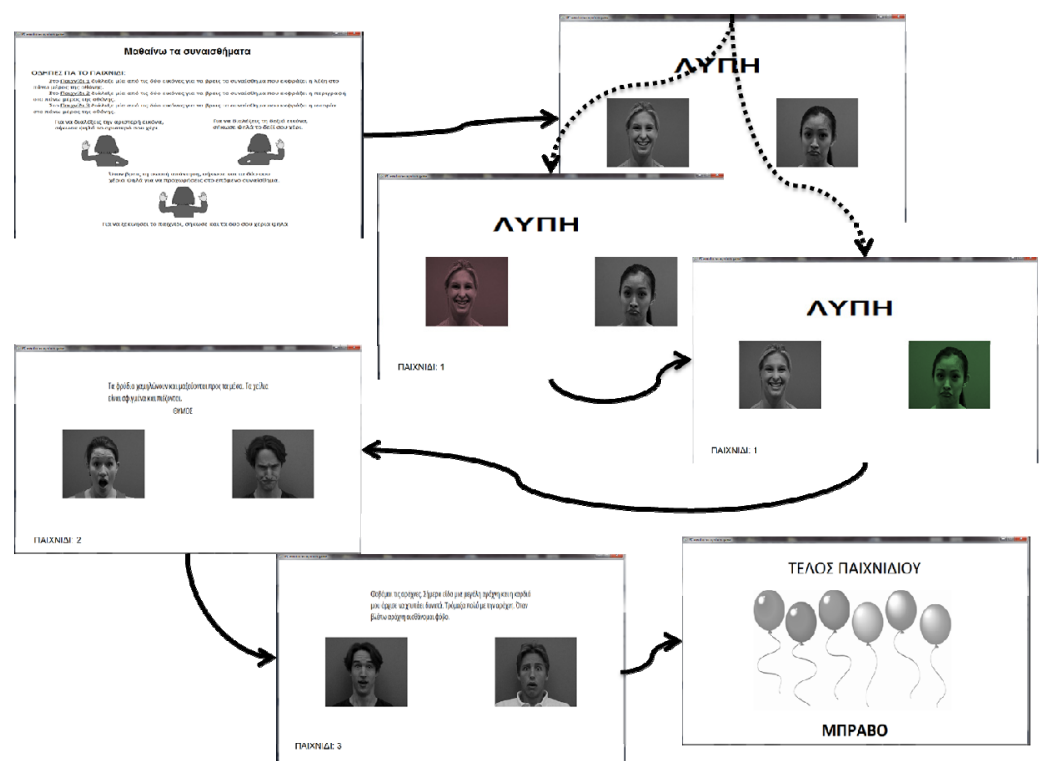

Fig. 4. Representative scenario [19]

\section{$5 \quad$ Conclusion and Future Work}

In this paper we have attempted to sketch the organizational underpinnings of the IOLAOS- a pilot effort aiming to build an open authorable framework for educational games for children by combining ludology and narratology. Our primary design target is to set up an operational model for carrying out the codification of educational theories and learning styles as well as the generation of ludic, narrative, and educational games according to needs, abilities and educational goals and to support this model with appropriate software platform and tools.

Ongoing work covers a variety of issues of both technological and educational engineering character. Some of the issues to be addressed in the immediate future include: (a) Elaborate on the Learning session compiler, (b) Further exploration of learning styles and educational theories in collaboration with expert and educator professional associations, (c) Run various use cases in vivo with the guidance and involvement of expert and educator professional associations (d) Enhance ludology aiming not only to children experience, but also to experts and teachers, and (e) Introduce further involvement of multimodal NUI devices so that the roles between game player and machine are reversed and the player performs gestures, sounds, grimaces etc. and the machine responds.

\section{References}

1. Prensky, M.: Fun, play and games: What makes games engaging. In: Digital Game-Based Learning, pp. 1-31. McGraw-Hill, New York (2001)

2. Huizinga, J.: Homo ludens: A study of the play-elements in culture. Routledge \& K. Paul, London (1949) 
3. Unity Game Engine. Unity Game Engine - Official Site, http : / unity3d. com (cited: February 5, 2014)

4. Hobson, P.R.: The autistic child's appraisal of expressions of emotion. JCPP 27, 321-342 (1986)

5. Rump, K.M., Giovannelli, J.L., Minshew, N.J., Strauss, M.S.: The development of emotion recognition in individuals with autism. Child Development 80, 1434-1447 (2009)

6. Bauminger, N.: The Facilitation of Social-Emotional Understanding and Social Interaction in High-Functioning Children with Autism: Intervention Out-comes. JADD 32, 283-298 (2002)

7. Faja, S., Aylward, E., Bernier, R., Dawson, G.: Becoming a face expert: A computerized face training program for high functioning individuals with autism spectrum disorders. Developmental Neuropsychology 33, 1-24 (2008)

8. Eikeseth, S.: Outcomes of comprehensive psycho-educational interventions for young children with autism. Research in Developmental Disabilities 30, 158-178 (2009)

9. Hwang, G.-J., Po-Han, W.: Advancements and trends in digital game-based learning research: a review of publications in selected journals from 2001 to 2010. British Journal of Educational Technology 43, E6-E10 (2012)

10. Resnick, M., Maloney, J., Monroy-Hernández, A., Rusk, N., Eastmond, E., Brennan, K., Millner, A., Rosenbaum, E., Silver, J., Silverman, B., Akafai, Y.: Scratch: programming for all. Commun. ACM 52, 60-67 (2009)

11. Monterrat, B., Lavoué, E., George, S.: Learning Game 2.0: Support for Game Modding as a Learning Activity. In: Proceedings of the 6th European Conference on Games Based Learning, pp. 340-347 (2012)

12. Lester, J.C., Jonathan, R.P., Bradford, M.W.: Narrative-Centered Learning Environments: A Story-Centric Approach to Educational Games. In: Emerging Technologies for the Classroom, pp. 223-237. Springer, New York (2013)

13. Padilla-Zea, N., Gutiérrez, F.L., López-Arcos, J.R., Abad-Arranz, A., Paderewski, P.: Mod-eling storytelling to be used in educational video games. Computers in Human Behavior (2013)

14. Wainer, A.L., Ingersoll, B.R.: The use of innovative computer technology for teaching social communication to individuals with autism spectrum disorders. Research in Autism Spectrum Disorders 5, 96-107 (2011)

15. Bernardini, S., Porayska-Pomsta, K., Smith, T.J.: ECHOES: An intelligent serious game for fostering social communication in children with autism. Information Sciences (2013) (in press)

16. Porayska-Pomsta, K., Anderson, K., Bernardini, S., Guldberg, K., Smith, T., Kossivaki, L., Hodgins, S., Lowe, I.: Building an Intelligent, Authorable Serious Game for Autistic Children and Their Carers. In: Reidsma, D., Katayose, H., Nijholt, A., et al. (eds.) ACE 2013. LNCS, vol. 8253, pp. 456-475. Springer, Heidelberg (2013)

17. Foster, M.E., et al.: Supporting children's social communication skills through interactive narratives with virtual characters. In: Proceedings of the International Conference on Multimedia. ACM (2010)

18. Castelhano, N., Silva, F., Rezende, M., Roque, L., Magalhães, L.: Ludic Content in Multisensory Stimulation Environments: An Exploratory Study about Practice in Portugal. Occupational therapy international (2013)

19. Christinaki, E., Vidakis, N., Triantafyllidis, G.: Facial expression recognition teaching to preschoolers with autism: a natural user interface approach. In: Proceedings of the 6th Balkan Conference in Informatics, pp. 141-148. ACM (2013) 